\title{
Exact Propagators for Soliton Potentials
}

\author{
Andrey M. PUPASOV and Boris F. SAMSONOV \\ Department of Physics, Tomsk State University, 36 Lenin Ave., 634050 Tomsk, Russia \\ E-mail: pupasov@phys.tsu.ru,samsonov@phys.tsu.ru
}

Received October 01, 2005, in final form November 21, 2005; Published online November 24, 2005

Original article is available at http://www.emis.de/journals/SIGMA/2005/Paper020/

\begin{abstract}
Using the method of Darboux transformations (or equivalently supersymmetric quantum mechanics) we obtain an explicit expression for the propagator for the onedimensional Schrödinger equation with a multi-soliton potential.
\end{abstract}

Key words: Darboux transformations; SUSY QM; soliton potentials; propagator

2000 Mathematics Subject Classification: 81Q05; 81Q60

\section{Introduction}

It is nowadays incontestable that ideas of supersymmetry play an important role in modern theoretical and mathematical physics. Supersymmetric quantum mechanics (SUSY QM), introduced by Witten [1] as a toy model in supersymmetric quantum field theory, is a very efficient tool for studying different properties of non-relativistic quantum systems. In particular, as it was recently shown [2] it may become an essential ingredient of complex quantum mechanics which is currently under development since it may "cure" such a "disease" of non-Hermitian Hamiltonians as their non-diagonalizability, and can remove spectral singularities from the continuous part of the spectrum. On the other hand, it is well known [3] that SUSY QM is basically equivalent to the method of Darboux transformations [4] well known in connection with soliton theory [5]. We mean by soliton potentials such potentials of the one-dimensional stationary Schrödinger equation from which soliton solutions of the Korteweg-de Vries equation may be constructed [5] and which are SUSY partners of the zero potential. They find an application in quantum field theory for describing processes where solitons may play an essential role [6].

It is not an exaggeration to say that everything in nature undergoes time-evolution. Therefore, one of the crucial questions of any physical theory is to describe how a physical phenomenon evolves with time. In quantum mechanics time dependence of the wave function may be described with the help of the propagator which is nothing but the evolution operator in coordinate representation. As far as we know, the first attempt to find the propagator for a multi-soliton potential is due to Jauslin [7]. His method is based on an integral formula which relates solutions of two Schrödinger equations whose Hamiltonians are SUSY partners. Unfortunately, this approach when applied to the Schrödinger equation leads to divergent integrals. Therefore the author found the propagator for the heat equation with the one-soliton potential. The Schrödinger equation may be considered as the heat equation with the imaginary time. In this respect the following question arises: whether or not the Jauslin's result after the replacement $t \rightarrow i t$ gives the propagator for the one-soliton potential? We want to stress that the answer to this question is not trivial since such a replacement at the level of the Jauslin's integral transformation leads to divergent integrals. Our analysis shows that the answer to this question is positive.

We would like to note that the problem of finding propagators is more difficult than solving the stationary Schrödinger equation for a time-independent Hamiltonian, since knowledge of 
the propagator allows us to solve the Cauchy problem for the non-stationary equation with the same Hamiltonian, but with an arbitrary initial condition. This is similar to the Green function of the stationary equation; knowing it allows us to write down a solution of the inhomogeneous equation with an arbitrary inhomogeneity. Recently a method has been proposed for finding the Green function for a SUSY partner Hamiltonian [8]. In this paper, using a particular example of soliton potentials, we show that the method of SUSY QM is very helpful for finding exact propagators for Hamiltonians related by SUSY (or equivalently Darboux) transformations. We would like to stress that although many aspects of multi-soliton potentials are well-studied in the literature a closed form of the propagator as far as we know has not yet been published.

To make the paper self-contained, first we briefly review the method we are using and give a general expression for the propagator (Section 2 and the beginning of Section 3), and then apply it for finding the propagator for the multi-soliton potential (Section 3). Finally, we apply our general result to the case of one- and two-soliton potentials (Section 4) and suggest a formula for $N$-soliton potential.

\section{Darboux transformations and soliton potentials}

In this section we review briefly the method of Darboux (SUSY) transformations $[5,9,10]$ Also we construct a differential (i.e. Darboux) transformation operator for obtaining solutions of the Schrödinger equation with a soliton potential from solutions of the free particle equation we need in the following sections.

Consider two one-dimensional Schrödinger equations

$$
\begin{array}{ll}
\left(h_{0}-E\right) \psi_{E}(x)=0, & h_{0}=-\partial_{x}^{2}+V_{0}(x), \\
\left(h_{1}-E\right) \varphi_{E}(x)=0, & h_{1}=-\partial_{x}^{2}+V_{1}(x) .
\end{array}
$$

Suppose one knows solutions to equation (1). Then solutions to equation (2) can be found by acting with the transformation operator (we denote it as $L$ ) on solutions to equation (1), $\varphi_{E}=L \psi_{E}$. The main relation defining $L$ is the intertwining relation

$$
L h_{0}=h_{1} L \text {. }
$$

An essential point of the method is the choice of the operator $L$. If we restrict $L$ to be a differential operator it becomes Darboux transformation operator (see e.g. [9]). In this case the potential $V_{1}$ cannot be arbitrary, and should be found together with the transformation operator from the intertwining relation (3). If $L$ is a first order differential operator, the result is well-known (see e.g. $[5,9,10]$ ):

$$
L=-\partial_{x}+w(x), \quad V_{1}=V_{0}-2 w^{\prime}(x),
$$

where

$$
w(x)=(\ln u)_{x}, \quad h_{0} u=\alpha u .
$$

Function $u=u(x)$ and parameter $\alpha$ are called transformation function and factorization constant respectively.

Since the procedure is independent on the initial Hamiltonian, it can be repeated as many times as one desires. So, we can take $h_{1}$ as the initial Hamiltonian for the next transformation step to get $h_{2}$ and so on. In this way one arrives at chains of transformations. It is remarkable that the resulting action of a chain may be expressed in terms of solutions of the initial equation 
only. If all factorization constants are different from each other, one obtains Crum-Krein [11] formulas

$$
\begin{aligned}
& \varphi_{E}=L \psi_{E}=W\left(u_{1}, u_{2}, \ldots, u_{N}, \psi_{E}\right) / W\left(u_{1}, u_{2}, \ldots, u_{N}\right) \\
& V_{N}=V_{0}-2\left[\log W\left(u_{1}, u_{2}, \ldots, u_{N}\right)\right]^{\prime \prime}
\end{aligned}
$$

Here and in the following the symbol $W$ denotes a Wronskian, and $u_{j}=u_{j}(x)$ is an eigenfunction of the initial Hamiltonian (transformation function)

$$
h_{0} u_{j}(x)=\alpha_{j} u_{j}(x), \quad j=1,2, \ldots, N .
$$

To get soliton potentials one starts with the zero initial potential, $V_{0}(x)=0$, and uses the following set of $N$ (which is supposed to be even) transformation functions [5, 9, 12]

$$
\begin{aligned}
& u_{2 j-1}(x)=\cosh \left(a_{2 j-1} x+b_{2 j-1}\right), \\
& u_{2 j}(x)=\sinh \left(a_{2 j} x+b_{2 j}\right), \quad j=1,2, \ldots, N / 2 .
\end{aligned}
$$

They are solutions to the Schrödinger equation with the zero potential corresponding to eigenvalues $E_{j}=-a_{j}^{2}<0$, which are just the points of the discrete spectrum of $h_{N}=-\partial_{x}^{2}+V_{N}(x)$. An orthonormal set of its discrete spectrum eigenfunctions is given by

$$
\varphi_{n}(x)=\left(\frac{a_{n}}{2} \prod_{j=1(j \neq n)}^{N}\left|a_{n}^{2}-a_{j}^{2}\right|\right)^{1 / 2} \frac{W^{(n)}\left(u_{1}, u_{2}, \ldots, u_{n-1}, u_{n+1}, \ldots, u_{N}\right)}{W\left(u_{1}, u_{2}, \ldots, u_{N}\right)} .
$$

Here $W^{(n)}\left(u_{1}, u_{2}, \ldots, u_{n-1}, u_{n+1}, \ldots, u_{N}\right)$ is the Wronskian of order $N-1$ obtained from the Wronskian $W\left(u_{1}, u_{2}, \ldots, u_{N}\right)$ by dropping the function $u_{n}$.

We also need the continuous spectrum eigenfunctions of $h_{N}$, which should be found by acting with the operator $L(4)$ on plane waves $\psi_{k}(x)=1 / \sqrt{2 \pi} \exp (-i k x), k \in \mathbb{R}$

$$
\begin{aligned}
& \varphi_{k}(x)=\frac{1}{\sqrt{\left(k^{2}+a_{1}^{2}\right)\left(k^{2}+a_{2}^{2}\right) \cdots\left(k^{2}+a_{N}^{2}\right)}} L \psi_{k}(x), \\
& E=k^{2}, \quad \alpha_{k}=-a_{k}^{2}, \quad k=1, \ldots, N .
\end{aligned}
$$

The set of functions $\left\{\varphi_{n}(x), n=1, \ldots, N\right\}$ and $\left\{\varphi_{k}(x), k \in \mathbb{R}\right\}$ forms a complete and orthonormal set in the Hilbert space of square integrable functions on the whole real line.

It is interesting to note that for particular values of the parameters $a_{j}$ a multi-soliton potential may have a shape of a multi-well potential thus presenting an example of a multi-well exactly solvable potential.

\section{Propagator for a multi-soliton potential}

We use the definition of the propagator $K\left(x, y ; t^{\prime}, t^{\prime \prime}\right)$ of the Schrödinger equation as the coordinate representation of the evolution operator (see e.g. [13]). If one knows solutions of the non-stationary Schrödinger equation with a time-independent potential, which form a complete and orthonormal set (for continuous subset, if present, normalization is understood in the sense of generalized functions) in the Hilbert space of square integrable functions on the whole real line, then the propagator is given by

$$
K\left(x, y ; t^{\prime}, t^{\prime \prime}\right)=\theta\left(t^{\prime}-t^{\prime \prime}\right)\left[\sum_{n=0}^{N} \psi_{n}^{*}\left(x, t^{\prime}\right) \psi_{n}\left(y, t^{\prime \prime}\right)+\int_{-\infty}^{\infty} d k \psi_{k}^{*}\left(x, t^{\prime}\right) \psi_{k}\left(y, t^{\prime \prime}\right)\right] .
$$


Here $\left\{\psi_{n}(x, t)\right\}$ is the discrete part of the basis and $\left\{\psi_{k}(x, t), k \in \mathbb{R}\right\}$ is the continuous one. Everywhere we shall assume $t^{\prime}>t^{\prime \prime}$ and drop the step function $\theta\left(t^{\prime}-t^{\prime \prime}\right)$.

According to this formula, the propagator has two contributions

$$
K\left(x, y ; t^{\prime}, t^{\prime \prime}\right)=K_{d}\left(x, y ; t^{\prime}, t^{\prime \prime}\right)+K_{c}\left(x, y ; t^{\prime}, t^{\prime \prime}\right) .
$$

The first term at the right hand side of formula (9), $K_{d}$, corresponds to the discrete part of the basis, and the second one, $K_{c}$, proceeds from the continuous part. Our crucial observation is that the method of Darboux transformations gives both the discrete and the continuous parts of the basis for the transformed equation if a complete set of eigenfunctions for the initial Hamiltonian is known.

For soliton potentials the discrete part is given by (7) and the continuous one is given by (8). Therefore

$$
K_{d N}=\sum_{n=0}^{N} \varphi_{n}^{*}\left(x, t^{\prime}\right) \varphi_{n}\left(y, t^{\prime \prime}\right), \quad K_{c N}=\int_{-\infty}^{\infty} d k \varphi_{k}^{*}\left(x, t^{\prime}\right) \varphi_{k}\left(y, t^{\prime \prime}\right),
$$

where the additional subscript $N$ labels the order of the Darboux transformation, which in our case coincides with the number of solitons.

We compute first the value $K_{c N}$. After inserting $\varphi_{k}(x)$ from (8) into (10) we interchange derivatives with the integrals over the momentum $k$. This allows us to present the contribution from the continuous spectrum as an action of the transformation operator $L$ on an integral

$$
\begin{aligned}
K_{c N}\left(x, y ; t^{\prime}, t^{\prime \prime}\right) & =L_{x} L_{y} \int_{-\infty}^{\infty} d k \frac{\psi_{k}^{*}\left(x, t^{\prime}\right) \psi_{k}\left(y, t^{\prime \prime}\right)}{\left(k^{2}+a_{1}^{2}\right)\left(k^{2}+a_{2}^{2}\right) \cdots\left(k^{2}+a_{N}^{2}\right)} \\
& =\frac{1}{2 \pi} L_{x} L_{y} \int_{-\infty}^{\infty} d k \frac{e^{i k(x-y)+i k^{2}\left(t^{\prime}-t^{\prime \prime}\right)}}{\left(k^{2}+a_{1}^{2}\right)\left(k^{2}+a_{2}^{2}\right) \cdots\left(k^{2}+a_{N}^{2}\right)} .
\end{aligned}
$$

Here $L_{x}$ is given by (4) and $L_{y}$ is obtained from $L_{x}$ by the replacement $x \rightarrow y$. The integral in (11) is calculated in Appendix. Thus, the contribution from the continuous part of the basis has the form:

$$
K_{c N}\left(x, y, t^{\prime}, t^{\prime \prime}\right)=\frac{1}{2 \pi} \sqrt{\frac{i t}{2}} L_{x} L_{y} \sum_{n=1}^{N}\left(\prod_{m \neq n}^{N} \frac{1}{\alpha_{n}-\alpha_{m}}\right) I\left(a_{n} \sqrt{\frac{i t}{2}}, \sqrt{\frac{i}{8 t}}(x-y)\right) .
$$

Here $t=t^{\prime}-t^{\prime \prime}$ and

$$
I(a, x)=\int_{-\infty}^{\infty} d p \frac{\mathrm{e}^{-2 p^{2}+4 p x}}{p^{2}+a^{2}}=\frac{\pi}{a} \mathrm{e}^{2 a^{2}} \operatorname{Re}\left[\mathrm{e}^{4 i x a} \operatorname{erfc}(a \sqrt{2}+i x \sqrt{2})\right] .
$$

To compute $K_{d N}$, we simply replace in (10) the discrete basis eigenfunctions $\varphi_{i}$ according to (7) and take into account their time dependence, which yields

$$
K_{d N}=\sum_{n=0}^{N}\left(\frac{a_{n}}{2} \prod_{j=1(j \neq n)}^{N}\left|a_{n}^{2}-a_{j}^{2}\right|\right) \frac{W^{(n)}(x) W^{(n)}(y)}{W(x) W(y)} \mathrm{e}^{i a_{n}^{2} t}, \quad t=t^{\prime}-t^{\prime \prime} .
$$

Thus, we see that the propagator for the multi-soliton potential is expressed in terms of the error function, derivative of the error function and solutions corresponding to the discrete part of the basis. In the next Section, we suggest a simpler formula for the propagator. 


\section{Particular cases}

Let us consider how expressions (12) and (14) lead to the propagators for the one- and two-soliton potentials.

The one-soliton potential

$$
V(x)=-2 a^{2} / \cosh ^{2}(a x)
$$

may be obtained from the zero potential with help of the first order Darboux transformation with the transformation function $u(x)=\cosh (a x)$. The only discrete spectrum eigenfunction is $\varphi_{0}(x)=(a / \sqrt{2}) / \cosh (a x)$.

After some simple calculations we obtain from (9), (12)-(14) the propagator for the Schrödinger equation with the one-soliton potential

$$
\begin{aligned}
& K_{1}\left(x, y ; t^{\prime \prime}, t^{\prime}\right)=\frac{1}{\sqrt{4 \pi i t}} \mathrm{e}^{\frac{i(x-y)^{2}}{4 t}}+\frac{a \mathrm{e}^{i a^{2} t}}{4 \cosh (a x) \cosh (a y)}\left[\operatorname{erf}_{+}(a)+\operatorname{erf}{ }_{-}(a)\right], \\
& t=t^{\prime}-t^{\prime \prime} .
\end{aligned}
$$

Here $\operatorname{erf}_{ \pm}(a)$ is the error function taken at a special value of the argument

$$
\operatorname{erf}_{ \pm}(a)=\operatorname{erf}\left[a \sqrt{i t} \pm \frac{i \sqrt{i}}{2 \sqrt{t}}(x-y)\right] .
$$

Now two observations are in order. (i) We state that up to the replacement $t \rightarrow i t$ our result is in the perfect agreement with that of Jauslin [7]. This fact justifies the Jauslin's procedure of regularization of corresponding divergent integral. And (ii) we see that the propagator (15) has two contributions. The first term coincides with the propagator for the free particle and, hence, the second one describes just the one-soliton perturbation of the free particle at the level of the propagator.

In case of the two-soliton potential the method of Jauslin becomes very involved, since it is not clear how corresponding integral may be calculated. In contrast, our formulas (12) and (14) give an explicit expression for the propagator. To use formula (14), we first calculate necessary Wronskians with the transformation functions (5), where for simplicity we choose $b_{1}=b_{2}=0$, i.e. $u_{1}(x)=\cosh \left(a_{1} x\right), u_{2}(x)=\sinh \left(a_{2} x\right)$ and then after some algebra we get the propagator for the two-soliton potential

$$
\begin{aligned}
K_{2}\left(x, y ; t^{\prime \prime}, t^{\prime}\right)= & \frac{1}{\sqrt{4 \pi i t}} \mathrm{e}^{\frac{i(x-y)^{2}}{4 t}} \\
& +\frac{a_{1}\left(a_{2}^{2}-a_{1}^{2}\right) \sinh \left(a_{2} x\right) \sinh \left(a_{2} y\right) \mathrm{e}^{i a_{1}^{2} t}}{4 W(x) W(y)}\left[\operatorname{erf}{ }_{+}\left(a_{1}\right)+\operatorname{erf}{ }_{-}\left(a_{1}\right)\right] \\
& +\frac{a_{2}\left(a_{2}^{2}-a_{1}^{2}\right) \cosh \left(a_{2} x\right) \cosh \left(a_{2} y\right) \mathrm{e}^{i a_{2}^{2} t}}{4 W(x) W(y)}\left[\operatorname{erf}_{+}\left(a_{2}\right)+\operatorname{erf}_{-}\left(a_{2}\right)\right], \\
t=t^{\prime}-t^{\prime \prime} . &
\end{aligned}
$$

Once again we see that the propagator for the two-soliton potential has a structure similar to that of the one-soliton potential. Both the propagator (15) and (16) have two contributions: the exponential part which is the free particle propagator and the part responsible for the soliton perturbation of the zero potential.

This result suggests us the form of the propagator of the general $N$-soliton potential as a sum of two contributions, namely the free particle propagator in the form of the exponential function 
and an $N$-soliton perturbation:

$$
\begin{aligned}
K_{N}\left(x, y ; t^{\prime \prime}, t^{\prime}\right)= & \frac{1}{\sqrt{4 \pi i t}} \mathrm{e}^{\frac{i(x-y)^{2}}{4 t}}+\sum_{n=0}^{N}\left(\frac{a_{n}}{4} \prod_{j=1(j \neq n)}^{N}\left|a_{n}^{2}-a_{j}^{2}\right|\right) \\
& \times \frac{W^{(n)}(x) W^{(n)}(y)}{W(x) W(y)} \mathrm{e}^{i a_{n}^{2} t}\left[\operatorname{erf}_{+}\left(a_{n}\right)+\operatorname{erf}_{-}\left(a_{k}\right)\right], \quad t=t^{\prime}-t^{\prime \prime} .
\end{aligned}
$$

We have checked this formula for $N=3,4$ by the direct substitution into the Schrödinger equation.

\section{Conclusion}

In this paper using the known approach based on supersymmetric quantum mechanics (or equivalently, the method of Darboux transformation) we construct propagators for multi-soliton potentials. In particular, we present explicit expressions for the case of one- and two-soliton potentials. While comparing these results with the ones previously published by Jauslin [7], we conclude that the transformation $t \leftrightarrow i t$ (Whick rotation) is justified for regularization of divergent integrals of a special type. Finally, we suggest an explicit expression for the propagator for the general multi-soliton potential.

\section{Acknowledgements}

This work is partially supported by the President Grant of Russia 1743.2003.2.

\section{A Appendix}

Here we calculate the integral

$$
\int_{-\infty}^{\infty} d k \frac{\mathrm{e}^{i k x+i k^{2} t}}{\left(k^{2}+a_{1}^{2}\right)\left(k^{2}+a_{2}^{2}\right) \cdots\left(k^{2}+a_{N}^{2}\right)} .
$$

The identity

$$
\prod_{n=1}^{N} \frac{1}{k^{2}+a_{n}^{2}}=\sum_{n=1}^{N}\left(\prod_{j=1, j \neq n}^{N} \frac{1}{a_{j}^{2}-a_{n}^{2}}\right) \frac{1}{k^{2}+a_{n}^{2}}
$$

reduces it to a sum of simpler integrals of the form:

$$
\int_{-\infty}^{\infty} d k \frac{\mathrm{e}^{i k x+i k^{2} t}}{k^{2}+a^{2}}=\frac{1}{2 i a} \int_{-\infty}^{\infty} d k \mathrm{e}^{i k x+i k^{2} t}\left(\frac{1}{k-i a}-\frac{1}{k+i a}\right) .
$$

One can find the value of the integral at the right hand side of this equation in [14]

$$
\int_{-\infty}^{\infty} d p \frac{\mathrm{e}^{-2 p^{2}+4 p x}}{p+i \alpha}=-i \pi \mathrm{e}^{2 \alpha(\alpha-2 i x)} \operatorname{erfc}[\sqrt{2}(\alpha-i x)]
$$

which leads just to formula (13) for the function

$$
I(a, x)=\int_{-\infty}^{\infty} d p \frac{\mathrm{e}^{-2 p^{2}+4 p x}}{p^{2}+a^{2}} .
$$


[1] Witten E., Dynamical breaking of supersymmetry, Nucl. Phys. B, 1981, V.188, 513-554.

[2] Samsonov B.F., SUSY transformations between diagonalizable and non-diagonalizable Hamiltonians, J. Phys. A: Math. Gen., 2005, V.38, L395-L403; quant-ph/0503075.

Samsonov B.F., Spectral singularities of non-Hermitian Hamiltonians and SUSY transformations, J. Phys. A: Math. Gen., 2005, V.38, L571-L579; quant-ph/0507085.

[3] Andrianov A.A., Borisov N.V., Ioffe M.V., Eides M.I., SUSY QM: new look at the equivalence of quantum systems, Theor. Math. Phys., 1984, V.61, 17-28.

[4] Darboux G., Leçons sur la théorie générale des surfaces et les applications géométriques du calcul infinitésimal, Deuxiéme partie, Paris, Gautier-Villar at Fils, 1889.

[5] Matveev V.B., Salle M.A., Darboux transformations and solitons, Berlin, Springer, 1991.

[6] Rajaraman R., Solitons and instantons, North Holland Publ., 1982.

[7] Jauslin H.R., Exact propagator and eigenfunctions for multistable models with arbitrarily prescribed $N$ lowest eigenvalues, J. Phys. A: Math. Gen., 1988, V.21, 2337-2350.

[8] Samsonov B.F., Sukumar C.V., Pupasov A.M., SUSY transformation of the Green function and a trace formula, J. Phys. A: Math. Gen., 2005, V.38, 7557-7565; quant-ph/0507160.

[9] Bagrov V.G., Samsonov B.F., Darboux transformations, factorization, supersymmetry in the one-dimensional quantum mechanics, Theor. Math. Phys., 1995, V.104, 356-367.

Bagrov V.G., Samsonov B.F., Darboux transformations in quantum mechanics, Phys. Part. Nucl., 1997, V.28, 374-397.

[10] Mielnik B., Rosas-Ortiz O., Factorization: little or great algorithm?, J. Phys. A: Math. Gen., 2004, V.43, 10007-10035.

Fernandez D.J.C., Fernandez-Garcia N., Higher-order supersymmetric quantum mechanics, AIP Conf. Proc., 2005, V.744, 236-273; quant-ph/0502098.

[11] Crum M., Associated Sturm-Liouville systems, Quart. J. Math. Oxford Ser. (2), 1955, V.6, 121-127.

Krein M.G., On a continual analogue of the Christoffel formula from the theory of orthogonal polynomials, Dokl. Akad. Nauk SSSR, 1957, V.113, 970-973.

[12] Sukumar C.V., Supersymmetry and potentials with bound states at arbitrary energies and multisolitons configurations, J. Phys. A: Math. Gen., 1986, V.19, 2297-2316.

Sukumar C.V., Supersymmetry and potentials with bound states at arbitrary energies, J. Phys. A: Math. Gen., 1987, V.20, 2461-2481.

[13] Cohen-Tannoudji C., Diu B., Laloë F., Mécanique quantique, tome 1, Paris, Hermann, 1977.

[14] Man'ko V.I., Chikhachev A.S., Integrals of motion and exact solutions of the problem of two dispersing delta-wells, JETP, 1998, V.86, 335-343. 